\title{
Brain-Derived Neurotrophic Factor in Chronic Periodontitis
}

\author{
Jôice Dias Corrêa, ${ }^{1}$ Daniele Sirineu Pereira, ${ }^{2}$ Mila Fernandes Moreira Madeira, ${ }^{1}$ \\ Celso Martins Queiroz-Junior, ${ }^{1}$ Danielle Glória Souza, ${ }^{3}$ Mauro Martins Teixeira, ${ }^{4}$ \\ José Eustáquio Costa, ${ }^{1}$ Antônio Lúcio Teixeira, ${ }^{5}$ and Tarcília Aparecida da Silva ${ }^{1}$ \\ ${ }^{1}$ Department of Oral Surgery and Pathology, School of Dentistry, Federal University of Minas Gerais, Antonio Carlos Avenue 6627, \\ 31.270-901 Belo Horizonte, MG, Brazil \\ ${ }^{2}$ Department of Physical Therapy, School of Physical Education, Physical Therapy and Occupational Therapy, \\ Federal University of Minas Gerais, Antonio Carlos Avenue 6627, 31.270-901 Belo Horizonte, MG, Brazil \\ ${ }^{3}$ Department of Microbiology, Biological Sciences Institute, Federal University of Minas Gerais, Antonio Carlos Avenue 6627, \\ 31.270-901 Belo Horizonte, MG, Brazil \\ ${ }^{4}$ Department of Biochemistry and Immunology, Biological Sciences Institute, Federal University of Minas Gerais, \\ Antonio Carlos Avenue 6627, 31.270-901 Belo Horizonte, MG, Brazil \\ ${ }^{5}$ Department of Clinical Medicine, Faculty of Medicine, Federal University of Minas Gerais, Professor Alfredo Balena Avenue 110, \\ 30130-100 Belo Horizonte, MG, Brazil
}

Correspondence should be addressed to Tarcília Aparecida da Silva; silva.tarcilia@gmail.com

Received 22 July 2014; Accepted 7 December 2014; Published 21 December 2014

Academic Editor: Elaine Hatanaka

Copyright (C) 2014 Jôice Dias Corrêa et al. This is an open access article distributed under the Creative Commons Attribution License, which permits unrestricted use, distribution, and reproduction in any medium, provided the original work is properly cited.

Brain-derived neurotrophic factor (BDNF) is a member of the neurotrophic factor family. Outside the nervous system, BDNF has been shown to be expressed in various nonneural tissues, such as periodontal ligament, dental pulp, and odontoblasts. Although a role for BDNF in periodontal regeneration has been suggested, a function for BDNF in periodontal disease has not yet been studied. The aim of this study was to analyze the BDNF levels in periodontal tissues of patients with chronic periodontitis (CP) and periodontally healthy controls (HC). All subjects were genotyped for the rs4923463 and rs6265 BDNF polymorphisms. Periodontal tissues were collected for ELISA, myeloperoxidase (MPO), and microscopic analysis from $28 \mathrm{CP}$ patients and $29 \mathrm{HC}$ subjects. BDNF levels were increased in $\mathrm{CP}$ patients compared to $\mathrm{HC}$ subjects. A negative correlation was observed when analyzing concentration of $\mathrm{BDNF}$ and IL-10 in inflamed periodontium. No differences in frequencies of BDNF genotypes between CP and HC subjects were observed. However, BDNF genotype GG was associated with increased levels of BDNF, TNF- $\alpha$, and CXCL10 in CP patients. In conclusion, BDNF seems to be associated with periodontal disease process, but the specific role of BDNF still needs to be clarified.

\section{Introduction}

Periodontal health can be described as "a dynamic state where the activity of proinflammatory/antimicrobial cytokines to control infection is optimally balanced by anti-inflammatory mechanisms to prevent unwarranted inflammation" [1]. In subjects susceptible to periodontal disease (PD), an imbalance of the inflammatory response results in excessive production of proinflammatory cytokines and the subsequent loss of periodontal attachment [2]. On the other hand, controlling mechanisms prevent excessive tissue damage [3]. Furthermore, the release of tissue regenerating factors may contribute to periodontal regeneration by regulating the function of periodontal ligament cells, endothelial cells, and cementoblasts [4]. In this setting, neurotrophin brain-derived neurotrophic factor (BDNF) has been reported to enhance periodontal tissue regeneration $[4,5]$. BDNF is a member of the neurotrophin family which is expressed by vascular endothelium and osteoblastic, immune, and neuronal cells [6]. BDNF is reported to be involved in the joint inflammatory process and its production is increased in response to proinflammatory cytokines [6].

Although a role for BDNF in periodontal regeneration has been proposed, no information is available concerning BDNF and periodontal disease. The aim of this study was 
to measure the levels of BDNF in periodontal tissues from patients with chronic periodontitis. The presence of polymorphisms rs6265 and rs4923463 of the BDNF gene and its correlation with inflammatory and clinical parameters were also assessed.

\section{Material and Methods}

2.1. Subjects and Sample Collection. Twenty-eight patients with CP, treated at the Periodontal Clinic, School of Dentistry, at Universidade Federal de Minas Gerais (UFMG, Brazil), were enrolled in this study. Periodontal tissue samples were obtained during periodontal surgery. Patients in this study met the following inclusion criteria: previous history of $\mathrm{CP}$, diagnosed according to previously described criteria: (1) exhibiting more than one tooth with probing depth higher than $5 \mathrm{~mm}$, (2) exhibiting more than two sites with clinical attachment loss deeper than $6 \mathrm{~mm}$, and (3) exhibiting lesions distributed in more than two teeth in each quadrant [7]. Patients who met the following criteria were excluded: (1) having a history of smoking, (2) use of antibiotic, (3) usage of anti-inflammatory and/or immunosuppressive medications during the 6 preceding months, and (4) a history of any systemic diseases (i.e., immunologic and autoimmune disorders, diabetes mellitus). The control group (HC) comprised 29 age and gender matched periodontally healthy patients enrolled for third molar removal surgery.

Periodontal examination was performed in both groups of patients, $\mathrm{CP}$ and $\mathrm{HC}$, at the initial visit to determine probing depth (PD), clinical attachment loss (CAL), and bleeding on probing (BOP). The BOP was considered positive if bleeding occurred within 30 seconds after probing [8]. Measurements were performed full-mouth at 6 sites per tooth (mesiobuccal, midbuccal, distobuccal, mesiolingual, midlingual, and distolingual). All the measurements were performed by the same examiner. At the time of the examination a peripheral blood sample was taken from each patient and processed for polymorphism determination.

Written informed consent was obtained from all patients. The study protocol was approved by the Institutional Ethics Committee (Protocol 324/08).

2.2. Inflammatory Infiltrate Evaluation. Periodontal tissue samples from periodontal pockets or healthy oral mucosa extracted during surgery of impacted third molars were fixed in $10 \%$ buffered formalin, embedded in paraffin wax, and cut longitudinally $(3 \mu \mathrm{m})$. The sections were deparaffinized, rehydrated, and stained with $H \& E$ for evaluation of the inflammatory infiltrate. Inflammatory cells were counted in four fields in two independent sections, using a light microscope (Axioskop 40 ZEISS; Carl Zeiss, Gottingen, Germany) at 400x magnification. Data were expressed as total inflammatory cells/field.

2.3. ELISA. The concentrations of the IL-17A, BDNF, IL10 , and TNF- $\alpha$ and the chemokine CXCL10 were measured in periodontal tissues by enzyme-linked immunosorbent assay (ELISA) using commercially available kits (R\&D Systems, Minneapolis, MN, USA). The assay was performed according to manufacturer's instructions. The wavelength of the microplate reader was $492 \mathrm{~nm}$. The lower limit
TABLE 1: Demographic and clinical features of the studied subjects.

\begin{tabular}{lccc}
\hline & HC $(N=29)$ & $\mathrm{CP}(N=28)$ & $P$ value \\
\hline Age (SD) & $41.21(8.4)$ & $44.4(8.5)$ & 0.25 \\
Gender (\% F) & 56.5 & 51.20 & 0.63 \\
PD (SD) & $2.60(0.7)$ & $4.46(0.9)^{*}$ & $<0.0001$ \\
CAL (SD) & $2.06(0.9)$ & $5.07(0.8)^{*}$ & $<0.0001$ \\
BOP (SD) & $5.7(0.6)$ & $31.6(2.2)^{*}$ & $<0.0001$ \\
\hline
\end{tabular}

HC: healthy controls; $\mathrm{CP}$ : chronic periodontitis; SD: standard deviation; PD: probing depth; CAL: clinical attachment loss; BOP: bleeding on probing (\% of sites).

${ }^{*}$ Significantly higher than control $\left(P<0.05, \chi^{2}\right.$ test or Student's $t$-test $)$.

of detection for each cytokine was $15 \mathrm{pg} / \mathrm{mL}, 3.9 \mathrm{pg} / \mathrm{mL}$, $5.5 \mathrm{pg} / \mathrm{mL}, 20 \mathrm{pg} / \mathrm{mL}$, and $4.5 \mathrm{pg} / \mathrm{mL}$, respectively, for IL-17A, IL-10, TNF- $\alpha$, BDNF, and CXCL10. The data were determined using a standard curve prepared for each assay and expressed as picograms of cytokine/chemokine per $100 \mathrm{mg}$ of tissue.

2.4. Myeloperoxidase. Periodontal tissue samples were also used for determination of myeloperoxidase (MPO) activity, a neutrophil enzyme marker, as described earlier [9]. The MPO activity in homogenized periodontal tissues was evaluated by enzymatic reaction and absorbance was measured at $450 \mathrm{~nm}$. The MPO content was expressed as relative units calculated from standard curves based on MPO activities from 5\% casein peritoneal-induced neutrophils assayed in parallel.

2.5. DNA Isolation and Genotyping Analysis. Total genomic DNA was extracted from blood samples using QIAamp DNA Blood Mini Kit (QIAGEN, Valencia, CA, USA) according to manufacturer's instructions. Quality, integrity, and quantity of DNA were analyzed by NanoDrop spectrophotometer (Thermo Scientific, Wilmington, DE, USA).

TaqMan genotyping assays were obtained from Applied Biosystems, Inc. (Foster City, CA, USA). The assay identification code for each respective SNP is BDNF rs6265 and rs4923463. All amplifications were carried out in an ABI $7900 \mathrm{H}$ thermal cycler (Applied Biosystems, Foster City, CA, USA) using TaqMan Genotyping Master Mix and following manufacturer's recommended amplification conditions.

2.6. Data Analysis. Data were expressed as mean \pm standard deviation (SD). Chi-square test analysis was used to test for deviation of genotype frequencies from Hardy-Weinberg equilibrium. The levels of cytokines in periodontal tissues and the frequency of gene polymorphisms were compared by the Student's $t$-test and chi-square tests. $P$ values $<0.05$ were considered statistically significant. All data were analyzed using SPSS 17 (SPSS Inc., Chicago, IL, USA).

\section{Results}

3.1. Clinical and Inflammatory Parameters. The sample included in the current study was composed by age and gender matched groups. The clinical features PD, CAL, and BOP were significantly higher in the $\mathrm{CP}$ than in the $\mathrm{HC}$ group $(P<0.0001)$ (Table 1$)$. The levels of IL-17A, CXCL10, 


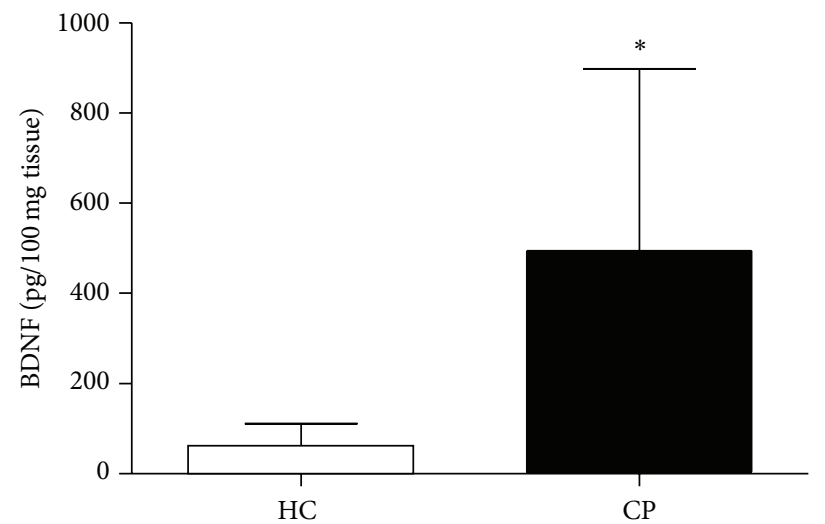

(a)

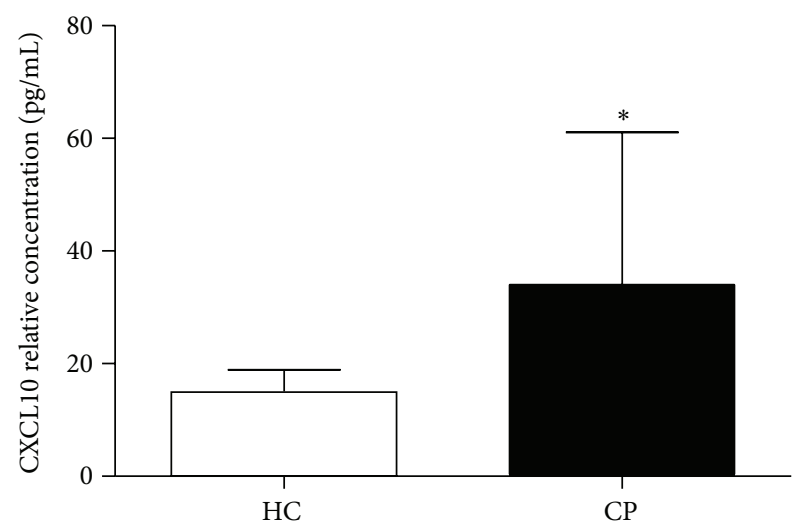

(c)

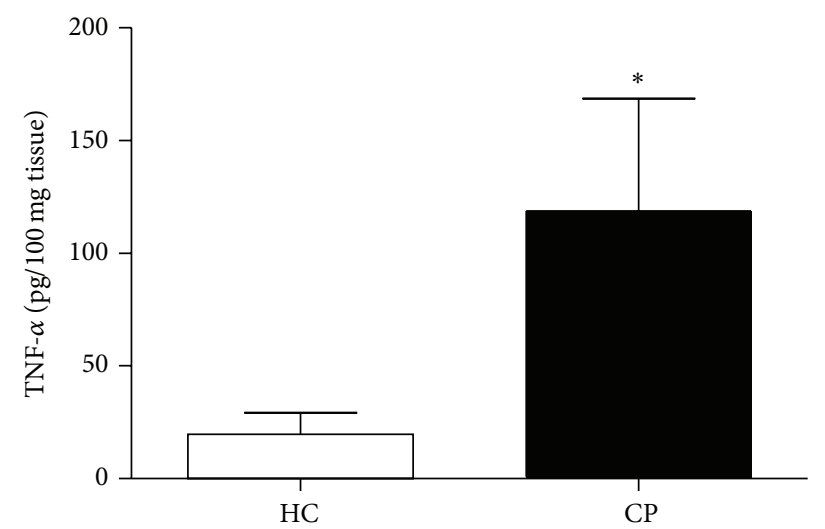

(b)

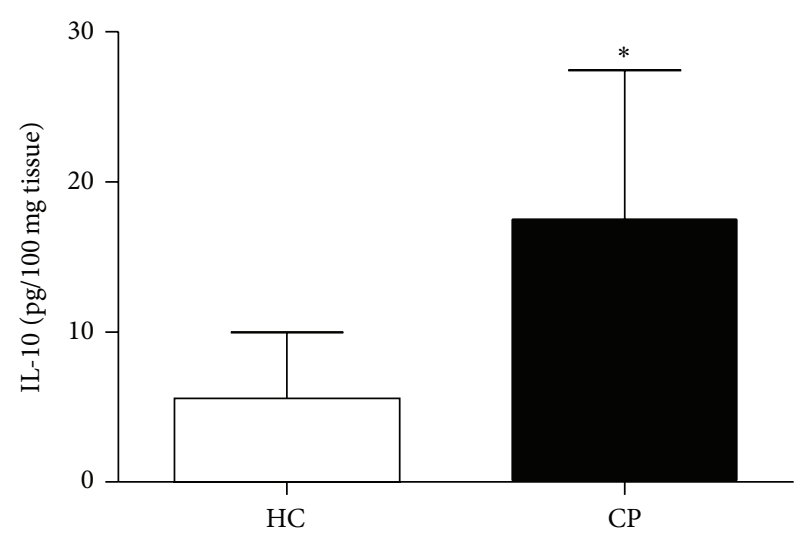

(d)

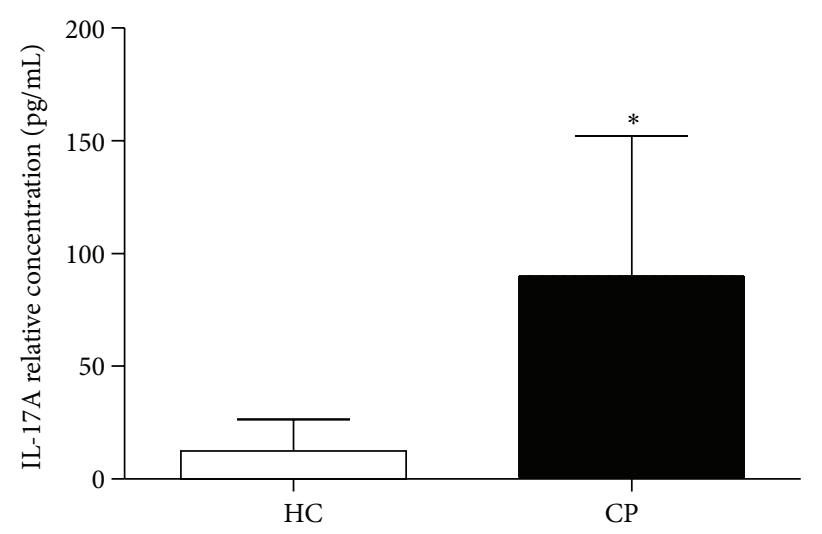

(e)

Figure 1: Levels of (a) BDNF, (b) TNF- $\alpha$, (c) CXCL10, (d) IL-10, and (e) IL17A in the periodontal tissues from CP and HC subjects. ${ }^{*}$ Statistically significant difference at $P<0.05$ in relation to HC (Student's $t$-test). HC: healthy control; CP: chronic periodontitis.

IL-10, TNF- $\alpha$, and BDNF in periodontal tissues were greater in CP patients than in controls (Figure 1). Moreover, the MPO activity and the inflammatory infiltrate in the periodontal tissues, characterized by polymorphonuclear and mononuclear leukocytes, were significantly higher in the $\mathrm{CP}$ than in the $\mathrm{HC}$ group (Figure 2).

The BDNF and IL-10 levels in periodontal tissues were negatively correlated $(R=-0.691, P=0.002)$, whereas no correlation between BDNF and IL-17A, TNF- $\alpha$, CXCL10, or clinical parameters was observed (PD, CAL, and BOP).

3.2. Association among Polymorphisms and Clinical Periodontal Parameters. Following the clinical investigation, the frequencies of polymorphisms (BDNF) were assessed in blood samples of HC and CP subjects (Table 2). The frequency of these genotypes agreed with the Hardy-Weinberg equilibrium 


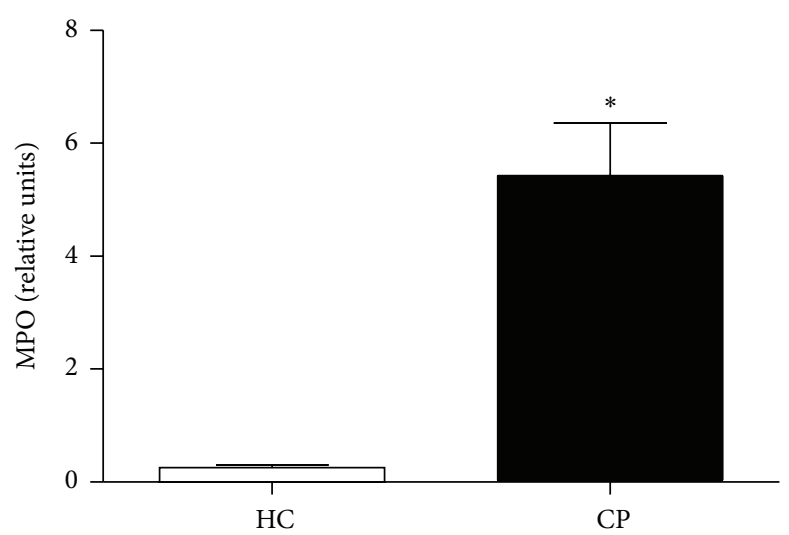

(a)

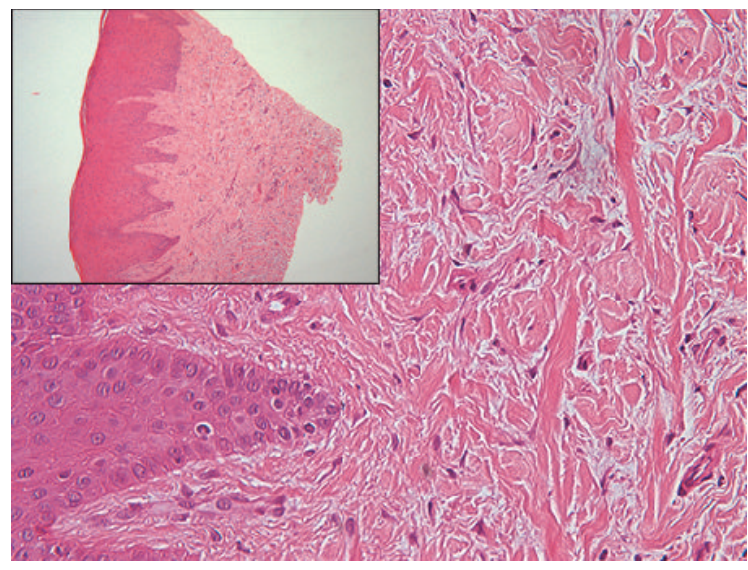

(c)

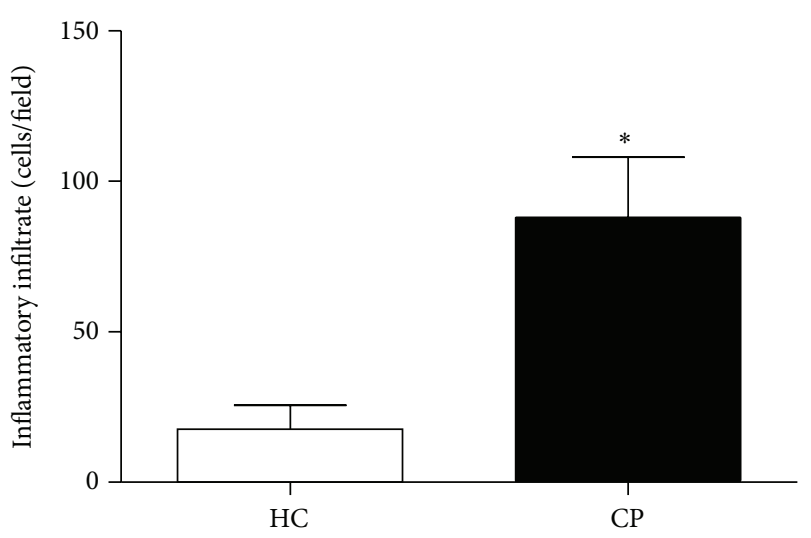

(b)

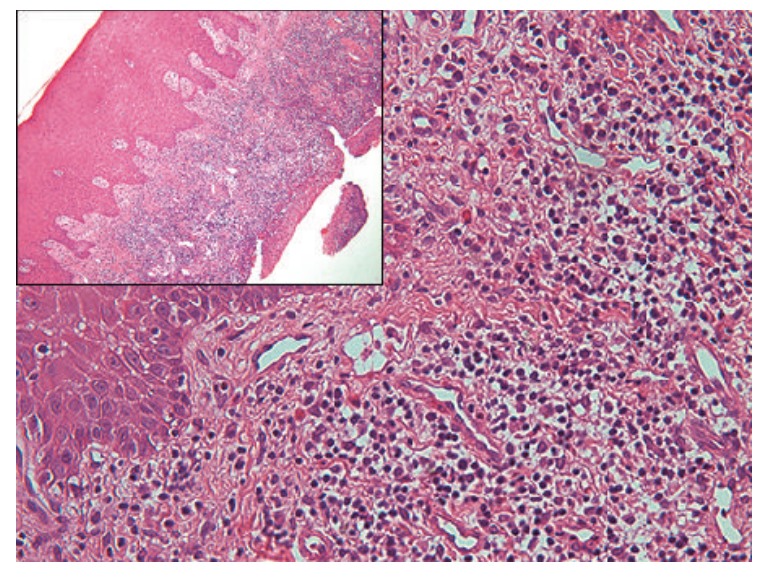

(d)

Figure 2: MPO activity (a). Number of inflammatory cells in the periodontal tissues from CP and HC subjects (b). Representative images of periodontal tissues from HC (c) and CP (d). H\&E staining. Magnification: 400x. Insert magnification: 100x. * Statistically significant difference at $P<0.05$ in relation to HC (Mann-Whitney $U$ test). HC: healthy control; CP: chronic periodontitis; MPO: myeloperoxidase.

TABLE 2: BDNF genotypes polymorphisms in patients with chronic periodontitis (CP) and healthy controls (HC).

\begin{tabular}{lcccc}
\hline Genotype & HC $(\%)$ & CP $(\%)$ & $P$ value & OR (95\% CI) \\
\hline BDNF rs6265 & & & & \\
GG & 88.88 & 83.33 & 0.15 & $0.30(0.05-1.67)$ \\
AG & 11.12 & 16.67 & 0.21 & \\
BDNF rs4923463 & & & & \\
AA & 88.88 & 76.00 & 0.44 & $0.50(0.08-3.01)$ \\
AG & 11.12 & 24.00 & 0.36 & \\
\hline
\end{tabular}

OR: odds ratio; CI: confidence interval.

$(P>0.05)$. The distribution of the BDNF polymorphisms was similar between the groups (Table 2).

We also investigated whether some of these polymorphisms were associated with worse clinical periodontal parameters. As shown in Table 3, no differences in clinical parameters were found when comparing the genotypes.

3.3. BDNF Genotypes Show Differences in the Expression of BDNF and Inflammatory Mediators in Periodontal Tissues.
The levels of BDNF and the inflammatory mediators CXCL10 and TNF- $\alpha$ were increased in GG genotype of BDNF rs6265 polymorphism (Figures 3(a), 3(b), and 3(c)), but MPO levels did not alter significantly (Figure 3(d)). In BDNF rs4923463 polymorphism the levels of BDNF and MPO did not differ, but the levels of CXCL10 and TNF- $\alpha$ were higher in patients with AA genotype (Figures 3(e)-3(h)).

\section{Discussion}

A wide range of nonneural cells in peripheral tissues or in the immune system expresses neurotrophins and their receptors. Thus, the mitogenic and immune regulatory functions of neurotrophins have been discussed $[6,10-12]$. The neurotrophin BDNF is reported to be involved in inflammatory reactions [13], and its production is increased in response to proinflammatory cytokines [14]. The present study is the first to demonstrate that BDNF levels were increased in periodontal tissues from chronic periodontitis compared to healthy subjects. In agreement with our findings, BDNF was found in high levels in the plasma of patients with osteoarthritis [15] and in patients with rheumatoid arthritis [6]. While some authors [15] reported that BDNF levels were 


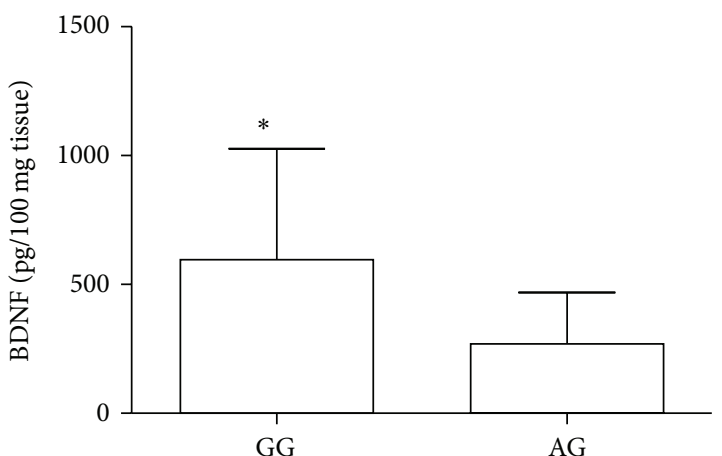

(a)

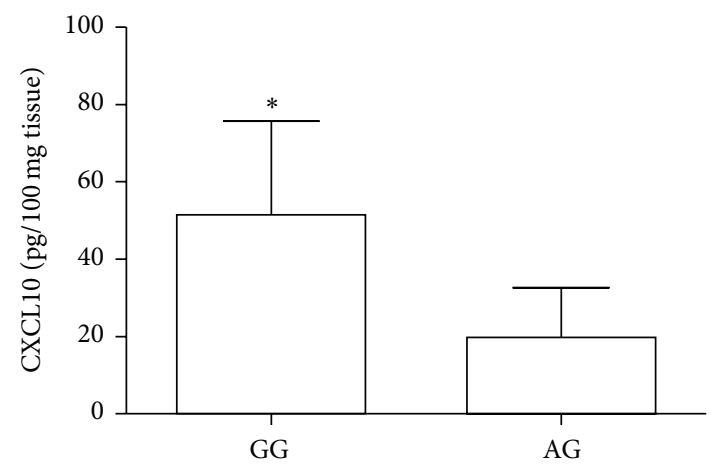

(c)

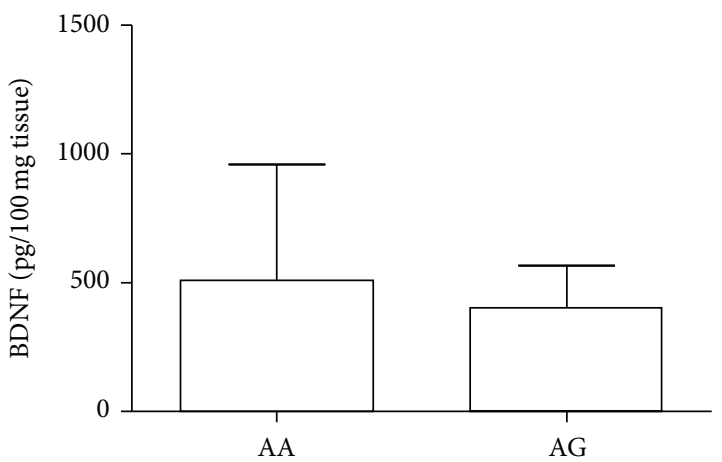

(e)

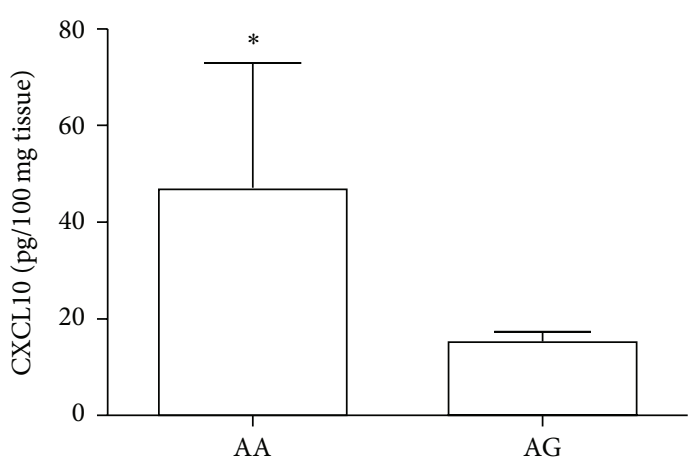

(g)

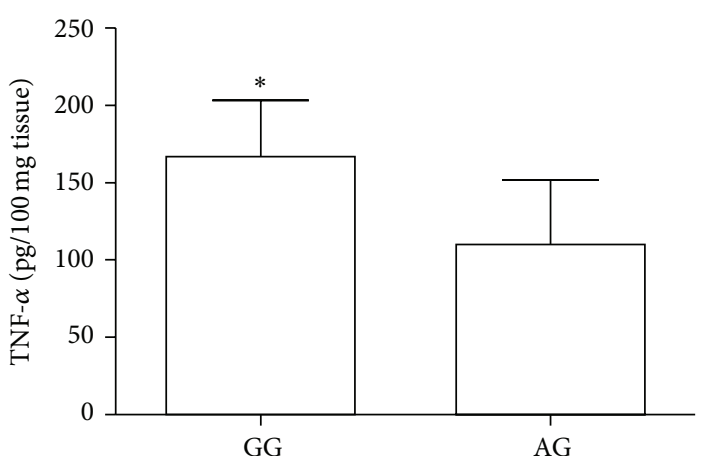

(b)

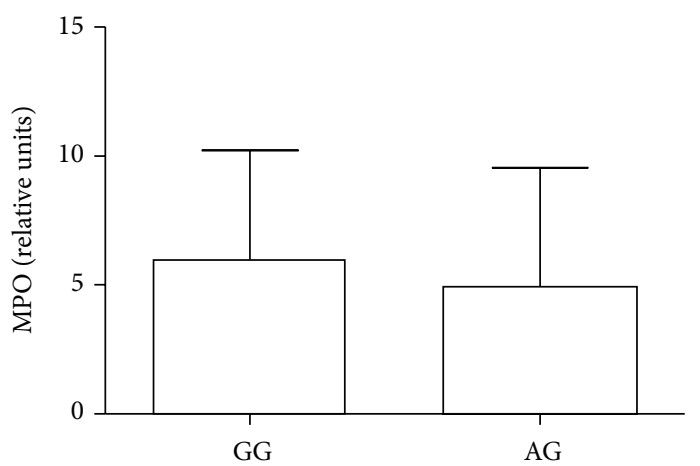

(d)

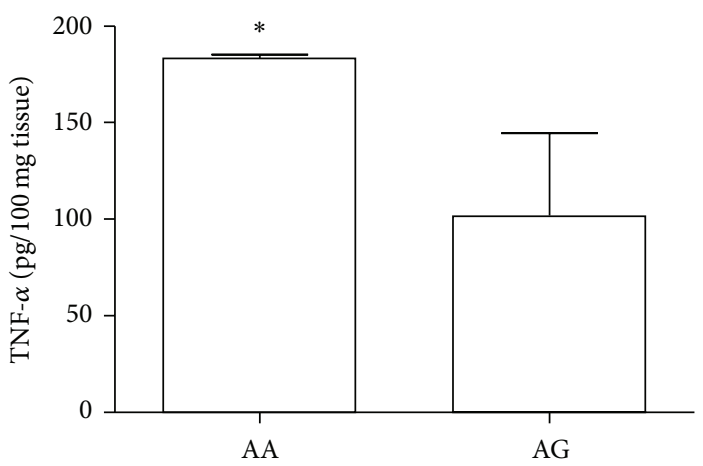

(f)

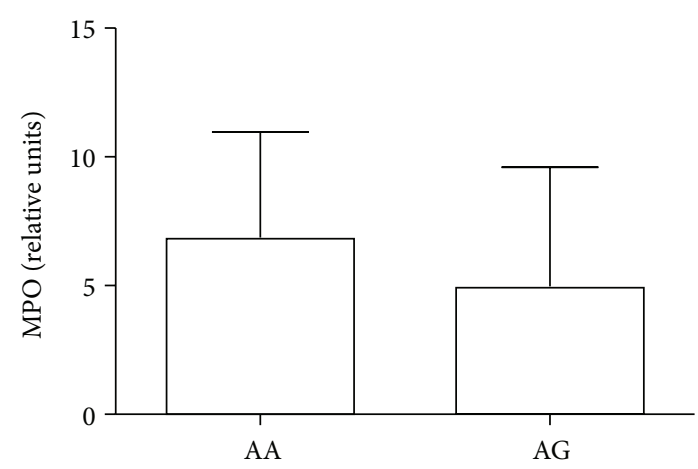

(h)

FIGURE 3: Levels of BDNF and inflammatory mediators in CP patients according to each BDNF genotype. (a) Levels of BDNF, (b) TNF- $\alpha$, and (c) CXCL10 and (d) MPO in periodontal tissues samples with BDNF rs6265 polymorphism. (e) Levels of BDNF, (f) TNF- $\alpha$, and (g) CXCL10 and (h) MPO in periodontal tissues samples with BDNF rs4923463 polymorphism. * Statistically significant difference at $P<0.05$ in comparison to the AG genotype (Student's $t$-test). 
TABLE 3: Association between BDNF polymorphisms and clinicopathological features of chronic periodontitis.

\begin{tabular}{|c|c|c|c|c|c|c|}
\hline Genotype & $\mathrm{PD}(\mathrm{mm})$ & $P$ value & CAL $(\mathrm{mm})$ & $P$ value & BOP (\%) & $P$ value \\
\hline \multicolumn{7}{|c|}{$B D N F$ rs6265 } \\
\hline GG & 4.58 & \multirow{2}{*}{0.586} & 5.42 & \multirow{2}{*}{0.744} & 30.80 & \multirow{2}{*}{0.115} \\
\hline $\mathrm{AG}$ & 4.35 & & 5.35 & & 22.46 & \\
\hline \multicolumn{7}{|c|}{$B D N F$ rs4923463 } \\
\hline AA & 4.46 & \multirow{2}{*}{0.729} & 5.21 & \multirow{2}{*}{0.734} & 30.81 & \multirow{2}{*}{0.323} \\
\hline $\mathrm{AG}$ & 4.60 & & 5.30 & & 25.37 & \\
\hline
\end{tabular}

significantly correlated with self-reported pain, others [6] did not find association between BDNF and clinical parameters of arthritis. We did not observe association of BDNF levels with clinical parameters.

Several studies analyzed BDNF rs6265 polymorphisms in psychiatric disorders [16-18]. To date, few studies examined the rs 4923463 polymorphism $[16,18-20]$. The rs 4923463 is adjacent to the functional polymorphism rs6265 Val66Met [19]. While one study found correlation between SNP rs4923463 and attention-deficit/hyperactivity disorder, schizophrenia, and risk of suicide in bipolar disorder, another study did not find any correlation between schizophrenia and rs4923463 polymorphism [18].

Previously, two studies have evaluated the effects of BDNF polymorphisms in bone [21, 22], but there are no available studies in periodontal disease. In the present study we did not find differences in BDNF genotype distribution between patients with $\mathrm{CP}$ and controls. Nevertheless, we found that subjects with GG (rs6265) genotype expressed higher levels of BDNF in periodontal tissues, in agreement with a previous report showing that BDNF-M66 variant alters intracellular trafficking and impairs BDNF secretion [23]. On the other hand, rs6265 polymorphism was not associated with production of BDNF by immune cells from multiple sclerosis patients [24]. Interestingly, the SNP rs6265 was reported as a phosSNP, which means this SNP regulates protein phosphorylation [22]. The SNP rs6265 affects substratekinase interaction between BDNF protein and CHEK2 kinase and regulates BDNF phosphorylation at site T62. Subjects AA genotype carriers exhibited lower bone mineral density compared to G carriers [22]. Specifically, BDNF-V66 (major allele $G$ at rs6265) transfection significantly increases expression of osteoblast specific markers (OPN, BMP2, and $A L P)$ and promotes osteoblast differentiation and maturation in cell culture [22]. An association of rs6265 with bone metabolism was also suggested in the largest meta-analysis involving 32,961 individuals of European and East Asian ancestry. The study showed that rs6265 was associated with femoral neck bone mineral density. They found that homozygous minor allele A carriers (AA) have significantly decreased BMD compared to major allele G carriers (GA and GG) [21].

It has been reported that BDNF is able to induce an increase in IL-10 expression [25]. However, a negative correlation between the production of BDNF and IL-10 was observed in samples from patients with periodontitis. IL10 can inhibit the release of proinflammatory cytokines from monocytes/macrophages and can therefore inhibit the lipopolysaccharide- and IFN- $\gamma$-induced secretion of inflammatory cytokines (e.g., TNF- $\alpha$, IL-1 $\beta$, IL-6, CXCL8, and others) [26]. In periodontal disease, IL-10 is thought to be associated with lower disease severity [27].

Previous studies demonstrated that BDNF induces periodontal tissue regeneration by activation of cementoblasts differentiation, vascular endothelial cell migration [4], and also has a positive effect on bone remodeling $[5,28]$. This data together suggested that BDNF has a role in bone remodeling and any change in this neurotrophin levels could have an impact in bone repair.

Finally, we observe that CP subjects with GG (rs6265) and AA (rs4923463) genotypes demonstrated increased levels of TNF- $\alpha$ and CXCL10. CXCL10 has several roles, such as chemoattraction of macrophages, T cells, NK cells, and dendritic cells [2]. Some of these cells are important for tissue repair [29]. In addition, previous studies showed that exposure to BDNF substantially and synergistically enhanced TNF$\alpha$ levels in vitro [30], and TNF- $\alpha$ preconditioning increased proliferation, mobilization, and osteogenic differentiation in vitro [31]. We can hypothesize that the concomitant increase of BDNF, TNF- $\alpha$, and CXCL10 in patients with the GG genotype may be an attempt of the host to induce periodontal healing. So, maybe if these patients were examined after periodontal treatment, they could display higher and better levels of tissue regeneration compared to patient who do not exhibit the GG genotype.

In conclusion, BDNF seems to be related to periodontal pathogenesis and also involved in tissue repair. The results obtained here provide a benchmark for future studies with a large cohort of patients to help strengthen and understand the influence of neurotrophins in periodontal disease.

\section{Conflict of Interests}

The authors declare that there is no conflict of interests regarding the publication of this paper.

\section{Acknowledgments}

This study was supported by CAPES, CNPq, and FAPEMIG.

\section{References}

[1] S. L. Gaffen and G. Hajishengallis, "A new inflammatory cytokine on the block: re-thinking periodontal disease and the Th1/Th2 paradigm in the context of Th17 cells and IL-17," Journal of Dental Research, vol. 87, no. 9, pp. 817-828, 2008. 
[2] T. A. Silva, G. P. Garlet, S. Y. Fukada, J. S. Silva, and F. Q. Cunha, "Chemokines in oral inflammatory diseases: apical periodontitis and periodontal disease," Journal of Dental Research, vol. 86, no. 4, pp. 306-319, 2007.

[3] W. Ouyang, S. Rutz, N. K. Crellin, P. A. Valdez, and S. G. Hymowitz, "Regulation and functions of the IL-10 family of cytokines in inflammation and disease," Annual Review of Immunology, vol. 29, pp. 71-109, 2011.

[4] K. Takeda, H. Shiba, N. Mizuno et al., "Brain-derived neurotrophic factor enhances periodontal tissue regeneration," Tissue Engineering, vol. 11, no. 9-10, pp. 1618-1629, 2005.

[5] R. Jimbo, N. Tovar, M. N. Janal et al., "The effect of brainderived neurotrophic factor on periodontal furcation defects," PLoS ONE, vol. 9, no. 1, Article ID e84845, 2014.

[6] O. Grimsholm, S. Rantapää-Dahlqvist, T. Dalén, and S. Forsgren, "BDNF in RA: downregulated in plasma following antiTNF treatment but no correlation with inflammatory parameters," Clinical Rheumatology, vol. 27, no. 10, pp. 1289-1297, 2008.

[7] G. C. Armitage, "Development of a classification system for periodontal diseases and conditions," Annals of Periodontology, vol. 4, no. 1, pp. 1-6, 1999.

[8] E. Newbrun, "Indices to measure gingival bleeding," Journal of Periodontology, vol. 67, no. 6, pp. 555-561, 1996.

[9] J. N. de Francischi, C. M. Queiroz-Junior, C. M. D. F. Pacheco, A. H. Fonseca, A. Klein, and M. V. Caliari, "Myeloperoxidase content is a marker of systemic inflammation in a chronic condition: the example given by the periodontal disease in rats," Mediators of Inflammation, vol. 2009, Article ID 760837, 7 pages, 2009.

[10] J. Dudás, M. Bitsche, V. Schartinger, C. Falkeis, G. M. Sprinzl, and H. Riechelmann, "Fibroblasts produce brain-derived neurotrophic factor and induce mesenchymal transition of oral tumor cells," Oral Oncology, vol. 47, no. 2, pp. 98-103, 2011.

[11] S. Forsgren, O. Grimsholm, T. Dalén, and S. RantapääDahlqvist, "Measurements in the blood of BDNF for RA patients and in response to anti-TNF treatment help us to clarify the magnitude of centrally related pain and to explain the relief of this pain upon treatment," International Journal of Inflammation, vol. 2011, Article ID 650685, 7 pages, 2011.

[12] P. B. Ehrhard, P. Erb, U. Graumann, and U. Otten, "Expression of nerve growth factor and nerve growth factor receptor tyrosine kinase Trk in activated CD4-positive T-cell clones," Proceedings of the National Academy of Sciences of the United States of America, vol. 90, no. 23, pp. 10984-10988, 1993.

[13] M. Kerschensteiner, E. Gallmeier, L. Behrens et al., "Activated human T cells, B cells, and monocytes produce brain-derived neurotrophic factor in vitro and in inflammatory brain lesions: a neuroprotective role of inflammation?" The Journal of Experimental Medicine, vol. 189, no. 5, pp. 865-870, 1999.

[14] L. Jornot, E. Grouzmann, J. S. Lacroix, and T. Rochat, "BDNF and DPP-IV in polyps and middle turbinates epithelial cells," Rhinology, vol. 45, no. 2, pp. 129-133, 2007.

[15] A. P. Simão, V. A. Mendonça, T. M. de Oliveira Almeida et al., "Involvement of BDNF in knee osteoarthritis: the relationship with inflammation and clinical parameters," Rheumatology International, vol. 34, no. 8, pp. 1153-1157, 2014.

[16] F. S. Neves, L. Malloy-Diniz, M. A. Romano-Silva et al., "The role of BDNF genetic polymorphisms in bipolar disorder with psychiatric comorbidities," Journal of Affective Disorders, vol. 131, no. 1-3, pp. 307-311, 2011.

[17] J. Herbert, M. Ban, G. W. Brown et al., "Interaction between the BDNF gene Val/66/Met polymorphism and morning cortisol levels as a predictor of depression in adult women," British Journal of Psychiatry, vol. 201, no. 4, pp. 313-319, 2012.

[18] A. Squassina, P. Piccardi, M. del Zompo et al., "NRG1 and BDNF genes in schizophrenia: an association study in an Italian casecontrol sample," Psychiatry Research, vol. 176, no. 1, pp. 82-84, 2010.

[19] M. Lanktree, A. Squassina, M. Krinsky et al., "Association study of brain-derived neurotrophic factor (BDNF) and LIN7 homolog (LIN-7) genes with adult attention-deficit/hvperactivity disorder," American Journal of Medical Genetics B: Neuropsychiatric Genetics, vol. 147, no. 6, pp. 945-951, 2008.

[20] T. Lencz, R. H. Lipsky, P. DeRosse, K. E. Burdick, J. M. Kane, and A. K. Malhotra, "Molecular differentiation of schizoaffective disorder from schizophrenia using BDNF haplotypes," British Journal of Psychiatry, vol. 194, no. 4, pp. 313-318, 2009.

[21] K. Estrada, U. Styrkarsdottir, E. Evangelou et al., "Genome-wide meta-analysis identifies 56 bone mineral density loci and reveals 14 loci associated with risk of fracture," Nature Genetics, vol. 44, no. 5, pp. 491-501, 2012.

[22] F.-Y. Deng, L.-J. Tan, H. Shen et al., "SNP rs6265 regulates protein phosphorylation and osteoblast differentiation and influences BMD in humans," Journal of Bone and Mineral Research, vol. 28, no. 12, pp. 2498-2507, 2013.

[23] Z. Y. Chen, P. D. Patel, G. Sant et al., "Variant brain-derived neurotrophic factor (BDNF) (Met66) alters the intracellular trafficking and activity-dependent secretion of wild-type BDNF in neurosecretory cells and cortical neurons," Journal of Neuroscience, vol. 24, no. 18, pp. 4401-4411, 2004.

[24] B. Weinstock-Guttman, R. Zivadinov, M. Tamaño-Blanco et al., "Immune cell BDNF secretion is associated with white matter volume in multiple sclerosis," Journal of Neuroimmunology, vol. 188, no. 1-2, pp. 167-174, 2007.

[25] Y. Jiang, N. Wei, J. Zhu et al., "Effects of brain-derived neurotrophic factor on local inflammation in experimental stroke of rat," Mediators of Inflammation, vol. 2010, Article ID 372423, 10 pages, 2010.

[26] D. F. Fiorentino, A. Zlotnik, T. R. Mosmann, M. Howard, and A. O'Garra, "IL-10 inhibits cytokine production by activated macrophages," The Journal of Immunology, vol. 147, no. 11, pp. 3815-3822, 1991.

[27] Q. Zhong, C. Ding, M. Wang, Y. Sun, and Y. Xu, "Interleukin10 gene polymorphisms and chronic/aggressive periodontitis susceptibility: a meta-analysis based on 14 case-control studies," Cytokine, vol. 60, no. 1, pp. 47-54, 2012.

[28] T. Yamashiro, T. Fukunaga, K. Yamashita, N. Kobashi, and T. Takano-Yamamoto, "Gene and protein expression of brainderived neurotrophic factor and TrkB in bone and cartilage," Bone, vol. 28, no. 4, pp. 404-409, 2001.

[29] S. K. Brancato and J. E. Albina, "Wound macrophages as key regulators of repair: origin, phenotype, and function," The American Journal of Pathology, vol. 178, no. 1, pp. 19-25, 2011.

[30] Y. S. Prakash, M. A. Thompson, and C. M. Pabelick, "Brainderived neurotrophic factor in TNF- $\alpha$ modulation of $\mathrm{Ca}^{2+}$ in human airway smooth muscle," American Journal of Respiratory Cell and Molecular Biology, vol. 41, no. 5, pp. 603-611, 2009.

[31] Z. Lu, G. Wang, C. R. Dunstan et al., "Activation and promotion of adipose stem cells by tumour necrosis factor-alpha preconditioning for bone regeneration," Journal of Cellular Physiology, vol. 228, no. 8, pp. 1737-1744, 2013. 


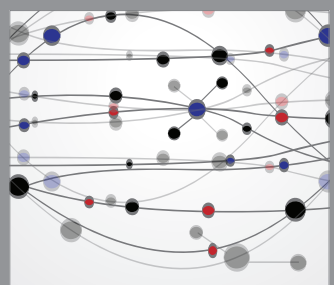

The Scientific World Journal
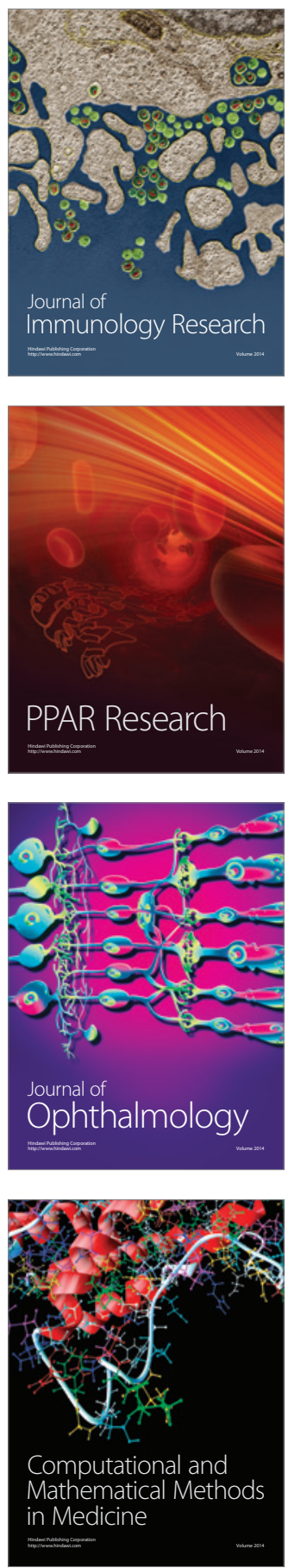

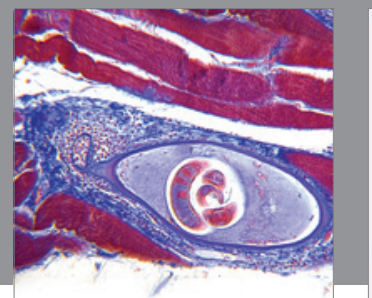

Gastroenterology

Research and Practice
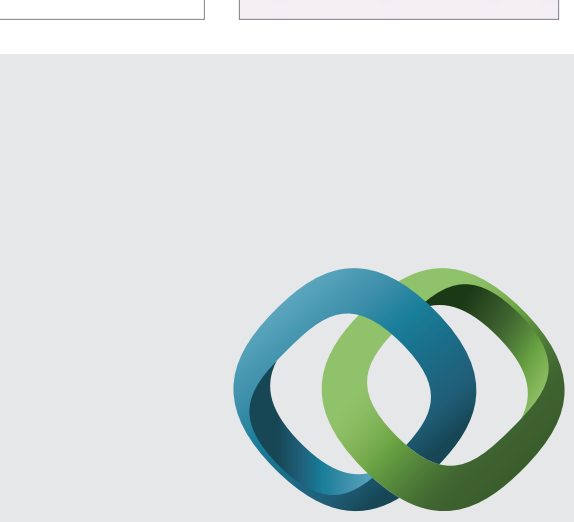

\section{Hindawi}

Submit your manuscripts at

http://www.hindawi.com
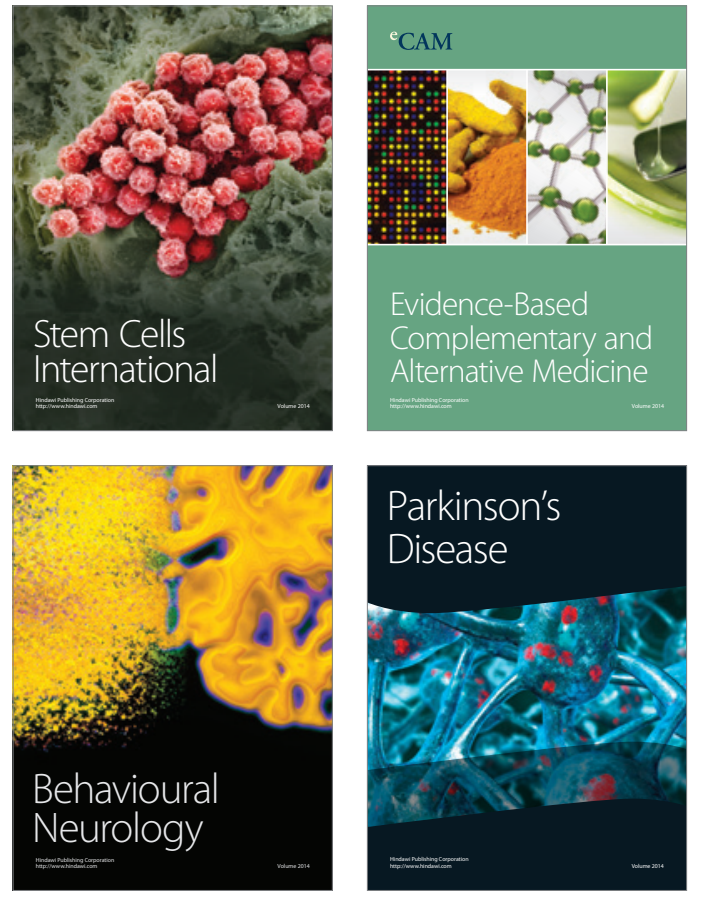
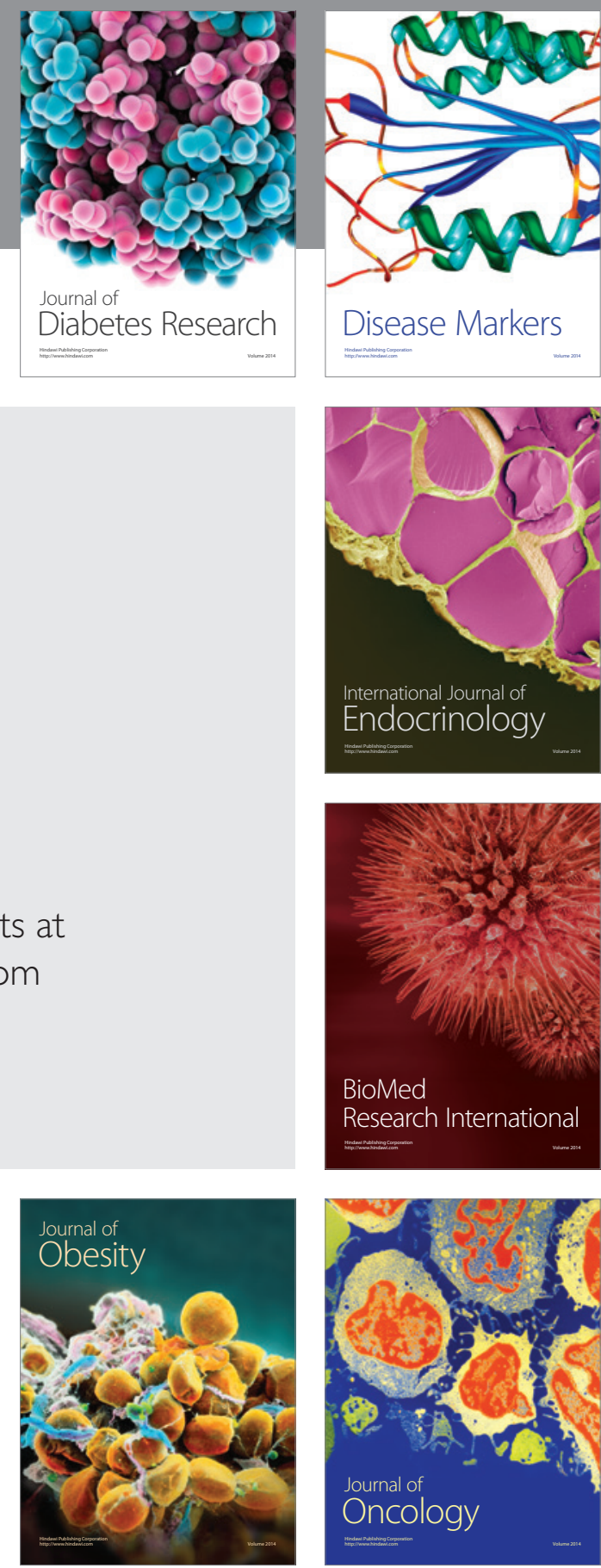

Disease Markers
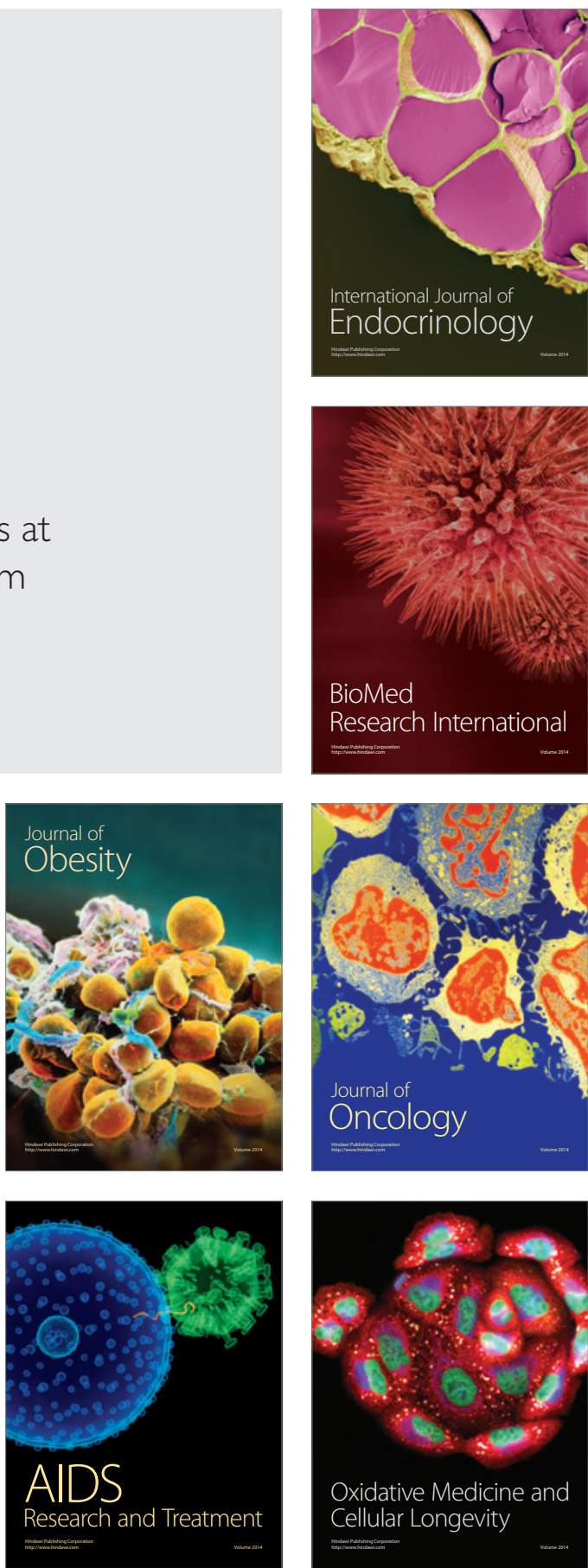